Questions vives

\section{Questions Vives}

Recherches en éducation

$\mathrm{N}^{\circ} 33 \mid 2020$

Visées heuristiques, praxéologiques et critiques dans les recherches participatives en éducation

\title{
Sur et pour... mais pas avec. Recherches doctorales sur le numérique en éducation
}

On and for... but not with. PhD on ICT in education.

\section{Jean-Luc Rinaudo}

\section{(2) OpenEdition}

\section{Journals}

Édition électronique

URL : http://journals.openedition.org/questionsvives/4583

DOI : $10.4000 /$ questionsvives. 4583

ISSN : 1775-433X

Éditeur

Université Aix-Marseille (AMU)

Édition imprimée

Date de publication : 15 décembre 2020

ISBN : 978-2-912643-57-5

ISSN : $1635-4079$

\section{Référence électronique}

Jean-Luc Rinaudo, «Sur et pour... mais pas avec. Recherches doctorales sur le numérique en éducation », Questions Vives [En ligne], №33 | 2020, mis en ligne le, consulté le 25 mars 2021. URL http://journals.openedition.org/questionsvives/4583; DOI : https://doi.org/10.4000/questionsvives 4583

Ce document a été généré automatiquement le 25 mars 2021.

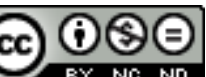

Questions Vives est mis à disposition selon les termes de la licence Creative Commons Attribution Pas d'Utilisation Commerciale - Pas de Modification 4.0 International. 


\section{Sur et pour... mais pas avec. Recherches doctorales sur le numérique en éducation}

On and for... but not with. PhD on ICT in education.

Jean-Luc Rinaudo

Lors de la conférence d'ouverture d'un colloque sur le numérique (Rinaudo, 2019), j'avais esquissé quelques éléments concernant les docteurs qualifiés par la $70^{\mathrm{e}}$ section $\mathrm{du}$ conseil national des universités (CNU) travaillant sur les technologies de l'information et de la communication (TIC). Cet article poursuit et amplifie cette première approche puisque l'objet de ce texte est de proposer des repères sur les perspectives de recherche adoptées par les docteurs ayant proposé un dossier de qualification aux fonctions de maitre de conférences à la section des sciences de l'éducation du $\mathrm{CNU}$, qui ont soutenu une thèse traitant du numérique en éducation et formation. La première partie de cet article expliquera la façon dont le corpus de données a été constitué. La partie suivante présentera les résultats de cette recherche avant qu'une discussion pointe des perspectives, en termes de recherches doctorales, où seront particulièrement étudiées les visées heuristique, critique et praxéologique des thèses du corpus.

\section{Constitution d'un corpus de données}

\subsection{Données générales}

Durant les cinq dernières années, entre 2015 et 2019, la $70^{\mathrm{e}}$ section du CNU a traité annuellement environ 300 dossiers de candidature aux fonctions de maitre de conférences. $45 \%$ des candidats en moyenne sont qualifiés. 
Tableau 1 : Dossiers qualification MCF $70^{e}$ section du CNU

\begin{tabular}{|l|l|l|l|}
\hline Années & Inscrits & Traités & Qualifiés \\
\hline 2015 & 377 & 292 & 121 \\
\hline 2016 & 390 & 310 & 154 \\
\hline 2017 & 352 & 270 & 129 \\
\hline 2018 & 356 & 284 & 127 \\
\hline 2019 & 364 & 288 & 125 \\
\hline TOTAL & 1839 & 1444 & 656 \\
\hline
\end{tabular}

3 Pour ce qui concerne plus spécifiquement les dossiers des candidats ayant soutenu un doctorat portant sur les technologies de l'information et de la communication (TIC) dans le champ de l'éducation, on constate le même taux de qualification ( $45 \%$ des dossiers étudiés relevant des TIC conduisent à une qualification). En outre, le nombre de dossiers relevant de ce domaine avoisine les $10 \%$ ce qui, compte tenu de la pluridisciplinarité caractéristique des sciences de l'éducation, est un nombre assez élevé.

Tableau 2 : Dossiers qualification $\mathrm{MCF} 70^{\mathrm{e}}$ section du CNU relevant des TIC

\begin{tabular}{|l|l|l|l|}
\hline Années & Inscrits TIC & Traités TIC & Qualifiés TIC \\
\hline 2015 & 34 & 27 & 8 \\
\hline 2016 & 36 & 32 & 18 \\
\hline 2017 & 34 & 26 & 11 \\
\hline 2018 & 33 & 29 & 11 \\
\hline 2019 & 30 & 24 & 15 \\
\hline Total & 167 & 138 & 63 \\
\hline
\end{tabular}

\subsection{Constitution du corpus}

4 Le premier choix dans la constitution du corpus de travail a été de considérer l'ensemble des dossiers déposés auprès de la 70 section du CNU entre 2015 et 2019 et non pas seulement ceux des docteurs qualifiés par cette section. D'une part, parce qu'une non qualification ne signifie pas nécessairement que la thèse soutenue n'est pas de bonne qualité, mais que plutôt les critères mis en œuvre par la $70^{\mathrm{e}}$ section pour les qualifications aux fonctions de maitre de conférences ne sont pas remplis, comme c'est souvent le cas en particulier avec l'exigence d'une publication dans une revue scientifique. D'autre part, parce que considérer l'ensemble des thèses des candidats, qualifiés comme non qualifiés, permet de quasiment doubler le nombre de dossiers auxquels on va s'intéresser et on peut espérer ainsi avoir un regard plus complet sur les 
démarches et les visées mobilisées par les jeunes chercheurs au cours de leur travail doctoral.

Le corpus de travail de cette recherche a été composé en plusieurs étapes. La première d'entre elles a consisté en un repérage, parmi les candidatures, de celles déposées par un docteur ayant soutenu une thèse portant sur les TIC. Pour ce faire, j'ai procédé en suivant trois phases. Tout d'abord, j'ai réalisé une recherche par mots clés sur les titres des thèses, à partir des termes: informatique, numérique, réseaux sociaux, TIC, technologies de l'information et de la communication, cyber-, virtuel, en ligne, FOAD, serious game, MOOC, simulation, e-learning. Bien sûr, les dérivés de ces mots clés (formes au singulier ou au pluriel, substantif ou adjectif) ont été pris en compte. Dans un deuxième temps, j'ai complété cette première liste à partir des thèses qui auraient échappé à ce premier filtre, à partir de la liste des directeurs de thèse qui avaient dirigé les thèses des premiers éléments du corpus retenus dans la première liste. Enfin, un troisième complément a été mis en œuvre à partir des rapporteurs de la $70^{\mathrm{e}}$ section du CNU spécialistes du numérique. En effet, chaque dossier se voit proposer, par le bureau de la $70^{e}$ section, au moins un rapporteur spécialiste du champ dans lequel la thèse a été soutenue. Pour les années 2015 à 2019, les rapporteurs recherchés ont été Brigitte Albero, Jacques Audran, Pierre-André Caron, Cédric Fluckiger, Philippe Gabriel, Alain Jaillet, Geneviève Lameul, Pascal Marquet, Ecaterina Pacurar, Jean-Luc Rinaudo, qui tous s'intéressent, peu ou prou, aux questions du numérique en éducation et en formation.

6 A partir de cette triple sélection, 144 dossiers ont été retenus. 23 dossiers ont été ôtés de cette liste, car ils constituaient des doublons, soit parce que la première demande n'avait pas conduit à une qualification, ce qui amenait les candidats à reformuler leur demande de qualification les années suivantes, soit parce que les candidats qui avaient été qualifiés en 2015 mais qui n'ayant pas obtenu un poste de maitre de conférences, demandaient de nouveau leur qualification en 2019.

7 L'étape suivante à consister à recueillir le résumé de ces 122 recherches doctorales sur le site thèses.fr qui recensent les thèses soutenues ou en cours en France. Ont dues être exclus du corpus, les 9 thèses soutenues à l'étranger et les 5 thèses qui ne comportaient pas de résumé. Enfin, 3 autres thèses portant sur la modélisation numérique de la croute terrestre, l'application sur les règles d'hygiène des soignants, la danse à l'ère du numérique, n'ont pas été retenues car, certes elles contenaient bien un des mots-clés, mais elles ont été considérées comme se trouvant hors du champ de la discipline par la $70^{\mathrm{e}}$ section du CNU.

$8 \mathrm{Au}$ final, le corpus se compose donc de 104 résumés de thèse et rassemble un peu plus de 30000 mots (31 192 exactement).

\subsection{Travailler sur des résumés}

9 Un travail sur des résumés semble pertinent dans le cadre de cette étude pour plusieurs raisons. D'une part, il était impossible de lire, dans le détail, plus d'une centaine de thèses pour cette recherche. De plus, je pars du postulat que les éléments importants de la thèse figurent dans le résumé, en particulier le type de démarche méthodologique mise en œuvre, l'accès au terrain s'il s'agit d'une recherche empirique, la perspective visée par la recherche, etc. 
10 Ce travail sur les résumés me semble plus pertinent pour cette étude qu'un travail à partir des seuls titres de thèse, à l'instar de ce qu'avaient réalisé Beillerot (1993) puis Beillerot et Demori (1998) qui eux travaillaient sur de grands corpus de plusieurs centaines de thèses. Le nombre de thèses retenues ici me semble relativement faible pour qu'à partir des seuls titres apparaissent des tendances sur les orientations des recherches doctorales. En revanche, notre postulat est que, comme l'affirmait Dessus (1999) dans son analyse des résumés aux Biennales de l'éducation, les préoccupations de recherche des jeunes docteurs peuvent être analysées à partir du lexique qu'ils utilisent dans leur résumé.

11 Le traitement des résumés, après mise en forme et anonymisation du corpus, a été réalisé à l'aide d'un logiciel de textométrie TXM (Heiden, Magué, Pincemin, 2010) qui présente l'avantage de n'effectuer que les traitements que l'utilisateur lui demande. Développé à l'École nationale supérieure de Lyon, ce logiciel open source établit, entre autres fonctionnalités, le lexique d'un corpus et calcule la fréquence d'utilisation d'un mot ou d'une expression. Il permet également de repérer le contexte d'emploi et de calculer les co-occurrences d'un terme. Contrairement à d'autres logiciels, TXM ne catégorise pas le corpus en unités sémantiques.

\subsection{Travailler sur le numérique}

12 Le secteur du numérique, qu'il y a encore quelques années on appelait technologies de l'information et de la communication et plus avant encore informatique, présente plusieurs intérêts pour ce travail sur les visées des recherches contemporaines. D'une part, les recherches dans ce domaine ne relèvent pas d'une seule orientation épistémologique. Ainsi, comme l'indique Baron (2013), ce domaine est pluridisciplinaire et en outre, la centration sur les niveaux d'enseignement (de l'école maternelle à l'enseignement supérieur) ou sur les entités considérées (apprenants, formateurs, dispositifs, outils, systèmes sociaux...) donne à ce domaine de recherche en éducation une focalisation plurielle qui s'appuie sur un large spectre de théories et de modèles.

13 Ainsi, ce domaine de recherche ne se présente pas de façon monolithique et est constitué par un ensemble d'approches (sociologie des usages, analyse systémique, psychologie des apprentissages, didactiques disciplinaires, analyse langagière, innovation, productions de ressources, etc.). De plus, les champs considérés par les docteurs relèvent des domaines scolaires, de la formation professionnelle, du champ des loisirs, de la santé, de la communication ou encore de la sphère du travail social. Ainsi on peut s'autoriser à penser qu'en étudiant les thèses portant sur le numérique, on peut se forger une idée de ce qui relève de l'ensemble des sciences de l'éducation, même si, on l'a bien compris, il ne s'agit en aucun cas d'en constituer un échantillon représentatif.

\subsection{Hypothèses de travail}

Les outils de lexicométrie permettent de repérer quels sont les mots employés dans les résumés. L'hypothèse que je propose est que si la recherche doctorale est conduite selon une démarche de recherche intervention, recherche action ou recherche collaborative, ces termes apparaitront explicitement dans les résumés. De même, la façon dont sont considérés les acteurs se fait jour à travers les emplois de leur 
désignation dans le corpus. Il s'agit donc ici de comprendre à partir des résumés de thèses, quelles visées poursuit le docteur dans sa recherche et selon quelle posture.

\section{Résultats}

\section{1. Éléments formels}

Parmi les 104 thèses retenues, 51 ont été soutenues en sciences de l'éducation, 21 en sciences de l'information et de la communication, 9 en sciences du langage. Puis viennent des thèses soutenues en psychologie (7) ou en sociologie (5). Le reste des thèses couvre un large champ des sciences humaines et sociales: arts, langues, économie, gestion, didactiques disciplinaires... On pourrait s'étonner du fait de ne pas retenir uniquement les thèses soutenues en sciences de l'éducation pour un tel article dans un dossier qui traite de cette discipline. Le choix qui a été fait de garder l'ensemble des thèses quelle que soit leur discipline se justifie par le fait que chaque docteur en proposant un dossier à la $70^{\mathrm{e}}$ section du CNU s'inscrit dans un projet de devenir un possible maitre de conférences en sciences de l'éducation.

Ces thèses ont été soutenues entre 2000 et 2018, mais il faut bien convenir que la plupart d'entre elles l'ont été sur la fin de la période considérée. Ceci est assez logique compte tenu du fait que le corpus a été constitué à partir des demandes de qualification au CNU entre 2015 et 2019. Ainsi, la moitié des 104 thèses ont été soutenues entre 2015 et 2018 et $83 \%$ entre 2013 et 2018 . On est donc en présence des travaux de recherche doctorale les plus récents, au moment de la constitution de ce corpus, en avril 2019.

\subsection{Lexique}

17 Le lexique utilisé par les docteurs dans les résumés de leur travail de thèse apporte quelques éléments relatifs à notre questionnement sur les visées des recherches sur le numérique.

Lorsqu'on étudie les mots pleins, c'est-à-dire les verbes, les substantifs, les adjectifs etc. mais pas les mots outils que constituent les déterminants, les prépositions, les pronoms ou les conjonctions, on repère des termes qu'on pouvait s'attendre à trouver en haute position hiérarchique, puisqu'ils sont liés à la composition même du corpus.

19 Ainsi un premier groupe de mots souvent utilisés dans les résumés étudiés pourraient probablement se retrouver dans l'étude de très nombreux résumés de thèses, quels qu'en soient les objets ou même les disciplines d'inscription. Il rassemble les termes désignant la recherche doctorale :

Tableau 3 : Lexique des résumés de thèse

\begin{tabular}{|l|l|l|}
\hline & Sing. & Pluriel \\
\hline recherche & 120 & 13 \\
\hline analyse & 97 & 17 \\
\hline travail & 83 & \\
\hline
\end{tabular}




\begin{tabular}{|l|l|l|}
\hline thèse & 81 & \\
\hline résultats & 4 & 69 \\
\hline étude & 68 & 24 \\
\hline
\end{tabular}

20 On repère également assez vite et sans surprise, un deuxième groupe de termes employés dans les résumés qui concerne le champ de l'éducation et de la formation. On notera au passage que le mot apprentissage est le mot plein le plus employé.

Tableau 4 : Lexique du champ de l'éducation et de la formation

\begin{tabular}{|l|l|l|}
\hline & Sing. & Pluriel \\
\hline apprentissage & 130 & 28 \\
\hline élèves & 17 & 109 \\
\hline formation & 77 & 5 \\
\hline enseignant.e.s & 21 & 75 \\
\hline pédagogiques & 29 & 44 \\
\hline éducation & & 42 \\
\hline enseignement & 3 & 39 \\
\hline
\end{tabular}

21 Enfin, toujours sans surprise, on trouve en position haute dans le classement hiérarchique du lexique, les termes relatifs au champ du numérique.

Tableau 5 : Lexique du numérique

\begin{tabular}{|l|l|l|}
\hline & Sing. & Pluriel \\
\hline numérique & 68 & 55 \\
\hline jeu & 60 & 15 \\
\hline utilisation & 52 & 4 \\
\hline usage & 51 & 48 \\
\hline information & 51 & 17 \\
\hline conception & 46 & 3 \\
\hline dispositif & 45 & 33 \\
\hline TIC & 43 & \\
\hline
\end{tabular}




\section{\begin{tabular}{l|l|l} 
ressources & 4 & 42
\end{tabular}} composition du corpus : des résumés de thèses sur le numérique (au sens large) dans le champ de l'éducation.

\subsection{Les visées de la thèse}

Au-delà du repérage des premiers mots pleins utilisés dans les résumés, ce qui nous intéresse ici concerne les enjeux et les visées de la recherche doctorale. Pour ce faire, les contextes d'emploi de certains termes issu lexique du corpus ont été étudiés.

Les termes démarche et méthodologie, que l'on compte respectivement 29 et 11 fois, sont souvent employés comme des synonymes, dans le corpus. Parfois d'ailleurs, les mêmes adjectifs les qualifient, notamment ceux qui permettent de spécifier l'orientation du recueil de données empiriques: qualitative, quantitative, mixte, comparative, expérimentale, constructiviste...

Le substantif approche, avec 46 occurrences, renvoie aux perspectives épistémologiques. Elles sont didactique, clinique, lexicométrique, documentaire... Ce terme est d'ailleurs là aussi souvent synonyme des deux précédents avec les qualificatifs empirique, quantitative, qualitative qui souvent l'accompagne. Deux emplois de ce mot interpellent plus particulièrement dans le cadre de ce texte, au sein d'un dossier sur les visées de la recherche. Il s'agit d'une revendication chez les docteurs d'une approche critique ou d'une approche praxéologique.

Pour l'approche critique, les docteurs dont référence à chaque fois à la sociologie critique (ou à la sociocritique). Il s'agit donc, pour les docteurs qui emploient ce terme, de désigner ou de s'inscrire dans un courant de recherche mais il ne s'agit sans doute pas ici d'une visée critique qui permettrait une prise de recul sur le questionnement, l'implication, le recueil de données, l'analyse conduite ou la restitution des résultats, c'est-à-dire à un critère de second niveau de recherche comme l'indiquait Beillerot, un travail au second degré qui ouvre une nécessaire "dimension de critique et de réflexivité »(Beillerot, 1991, p. 21).

Quant à l'approche praxéologique, l'expression est clairement employée en référence aux travaux d'anthropologie du didactique, notamment en didactique des mathématiques (Chevallard, 1998) et ici encore renvoie à un paradigme de recherche.

Le terme modèle et ses dérivés n'apportent guère d'éléments pour notre travail lorsqu'il s'agit d'indiquer des éléments de contexte de la thèse, par exemple les formes traditionnelles du modèle scolaire. L'étude des contextes d'emploi de ce terme montre une visée transformatrice des pratiques, notamment de conception des outils, avec la production d'un modèle de conception de document en e-learning ou la définition d'un modèle « unique et holistique » de conception des serious games. L'idée sous-jacente est souvent que l'utilisation d'un modèle théorique existant ou mieux encore la réalisation d'une nouvelle modélisation («nous pouvons ainsi présenter un modèle théorique général du concept d'appropriation d'une nouvelle ressource») permettra un changement comme par exemple «d'apporter des pistes pour améliorer 
l'appropriation du dispositif » ou encore « nous proposons des modèles visant à assister les concepteurs dans la création la mutualisation et la réutilisation de solutions ».

Dans les résumés du corpus, le mot intention ne porte pas sur celle du chercheur mais sur celle des acteurs étudiés. Par exemple : « la plupart des utilisateurs suivent le cours avec l'intention d'en réinvestir le contenu dans leur vie personnelle ou professionnelle».

30 L'emploi du mot enjeu, au singulier comme au pluriel, est le plus souvent réalisé pour traiter des domaines que la thèse permet d'aborder : enjeux économiques, éducatifs, culturels, institutionnels ou politiques sont ainsi cités.

31 Les 62 emplois des mots de la famille d'interaction concernent, le plus souvent, le dispositif technologique étudié, comme le tableau numérique interactif, ou encore le paradigme scientifique dans lequel se situe l'auteur comme l'interactionnisme symbolique. Lorsqu'il s'agit d'interactions entre des acteurs, comme pour «les interactions tuteurs apprenants ", il n'est jamais question d'une interaction avec le chercheur lui-même.

Pour repérer si certaines recherches étaient affichées comme des recherches-actions, des recherches-interventions, des recherches-accompagnement ou des recherchescollaboratives une attention a été portées sur ces termes.

L'expression recherche-action est utilisée dans deux résumés.

Dans le premier, il s'agit d'indiquer dans quelle perspective est conduite la thèse : « sur le plan de la méthode nous avons choisi de réaliser une recherche-action». La démarche menée dans cette thèse inscrite dans le champ du soin s'apparente à une recherche clinique dans le domaine de la santé : questionnaire initial qui conduit à la mise en place d'une pratique de soin à distance pour quelques cas dont l'analyse permet de proposer de nouvelles perspectives. Il s'agit d'une recherche-action dans la mesure où les protocoles et les hypothèses de la recherche ne sont pas construits en amont mais s'élaborent en fonction des premiers résultats empiriques de la recherche.

Le second, «les deux dernières études s'apparentent à des recherches-action visant à amener les étudiants à utiliser l'exerciseur en ligne à partir d'interventions pédagogiques réalisées en travaux dirigés ", apporte peu d'éléments qui permettent de comprendre en quoi la recherche menée est une recherche-action, notamment parce que le résumé ne permet pas de comprendre comment s'ajustent les interventions pédagogiques aux pratiques des étudiants. On pointe probablement là une limite au travail à partir de résumés. Il montre en revanche une volonté de transformation des pratiques des étudiants.

36 Si le mot intervention est peu employé ( 6 fois seulement au singulier ou au pluriel) et essentiellement pour désigner les actions des enseignants ou des formateurs, un emploi concerne une recherche-intervention dans laquelle l'auteur indique que l'objet de la thèse est la conception d'une formation en ligne et, qu'après la mise en place d'un premier dispositif, ont été réinvesties "les premières considérations issues des pratiques des apprenants au sein du dispositif dans ses conceptions ultérieures». Il semble que l'auteur se situe davantage dans une posture de conduite du changement que dans celle d'un accompagnement du changement (Bedin, 2013).

37 Le mot accompagnement, lui aussi peu utilisé (6 occurrences) renvoie le plus souvent aux besoins des apprenants en termes de formation: «le tuteur est principalement perçu dans un rôle d'accompagnement ». La forme verbale permet de repérer un emploi dans 
le contexte de l'accompagnement du changement «nos résultats permettent alors d'appuyer des propositions pour accompagner un changement dans les pratiques des formateurs ». Notons au passage que le mot changement (15 occurrences) est utilisé pour désigner les contextes évolutifs des terrains sur lesquelles se conduisent les thèses, "les universités sont en changement", ou plus fréquemment les transformations qu'entrainent les technologies numériques: "l'accélération des changements techniques et l'intégration des TIC dans la vie quotidienne renouvellent la problématique de la formation à l'ère du numérique: il est largement admis aujourd'hui que les technologies transforment les pratiques sociales à un rythme exponentiel ».

Quant aux 17 mots de la famille de collaboration, ils sont toujours utilisés pour spécifier des démarches de conception d'outils ou d'apprentissage, mais en aucun cas pour indiquer une recherche collaborative.

\subsection{Les acteurs}

Dans cette dernière partie de l'analyse, on cherchera comment sont désignés les acteurs de la recherche et quels sont les contextes d'emplois des termes employés de façon à repérer si le docteur s'inscrit dans une recherche collaborative avec les acteurs des terrains qu'il investigue.

Le terme acteur (12 emplois) renvoie exclusivement aux sujets de la recherche mais ne désigne jamais le chercheur. Les termes interactions et enjeux que nous avons étudiés plus avant, mais aussi positionnement et apprenants sont les termes calculés comme cooccurents du mot acteur (qui apparaissent en proximité au sein du corpus) mais pas le mot chercheur. Dans l'ensemble du corpus les emplois de ce dernier mot ne désignent pas spécifiquement l'auteur de la recherche à 6 reprises sur 9, comme dans la phrase suivante « la question des usages des TIC par les élèves suscite un intérêt particulier auprès des chercheurs de certaines disciplines ». On trouve les 3 autres emplois du mot chercheur dans deux résumés. L'un désigne le fait que le chercheur a été accueilli pour conduire plusieurs séquences d'observation dans des classes. L'autre indique, par deux emplois, que l'implication de la praticienne chercheure est prise en compte dans l'analyse des résultats. C'est d'ailleurs une des rares observations que l'on peut repérer d'une visée critique de la production scientifique, avec une analyse de la posture et de la distance à l'objet de recherche.

41 On peut faire le même constat avec les plus nombreux usages (95) des termes enseignant.e.s. Lorsqu'une interaction est pointée pour ces derniers, il s'agit d'une interaction avec les élèves ou leurs parents mais pas avec le chercheur. Les mêmes remarques valent pour professeur.e.s (18 emplois). Le mot professionnel.le.s également fortement employé (80 occurrences) est utilisé comme qualificatif (didactique professionnelle, développement professionnel, identité professionnelle, etc.) mais ne désigne jamais les acteurs, dans les résumés du corpus.

\section{Une visée pragmatique des recherches}

La lecture des résumés des thèses retenues pour la constitution de ce corpus étayée par l'analyse logicielle du lexique montre que la perspective heuristique est assumée ouvertement par de nombreux jeunes docteurs. La phrase issue d'un des 
résumés » contribuer à la connaissance de l'activité et des modalités d'apprentissage » me semble bien illustrer cette perspective heuristique à l'œuvre dans les travaux de thèse. Ce premier constat peut sembler assez logique dans la mesure où un travail doctoral doit être un travail de production de savoir savant, qui ici vise à éclairer, à donner du sens aux usages, aux pratiques, aux conceptions d'outils numériques dans le champ de l'éducation et de la formation.

La dimension critique semble moins abordée. Du moins, est-elle moins clairement revendiquée dans les résumés des thèses, à l'exception, dans un seul résumé, de l'analyse de l'implication du chercheur déjà mentionnée. Là encore, il s'agit probablement d'une limite à un travail sur les résumés dans lesquels la réflexion critique sur le mode de recueil de données, sur leur analyse, sur la question de la distance à l'objet ou au terrain de recherche comme sur celle de la restitution, est le plus souvent absente, même si elle est abordée dans le texte même de la thèse.

La plus grande surprise de ce travail est le fait que la dimension praxéologique est peu présente au sein des résumés. On peut repérer des résumés de thèse dans lesquels le docteur se montre soucieux d'articuler théorie et pratique. Cette préoccupation n'est pas nouvelle et peut rappeler les positions de Durkheim (1922) sur la pédagogie et la science de l'éducation, aux débuts du $\mathrm{XX}^{\mathrm{e}}$ siècle. Cependant la préoccupation de production de savoir semble première comme semble le montrer cet extrait qui décrit les buts de la thèse ordonnés de façon hiérarchique: " 1 un but épistémique par la production de concepts sur l'activité humaine et 2 un but transformatif par la conception d'aides favorisant des transformations majorantes ». Certes, il existe bien, et cela est repérable dans les résumés, une intention chez les chercheurs de " combler l'écart qui existe entre la théorie et la pratique, entre la science et l'action » (SaintArnaud, Mandeville, Bellemare, 2002, p. 30). En revanche, la dimension "d'autonomisation et de conscientisation de l'agir (à tous les niveaux d'interaction sociale) dans son histoire, dans ses pratiques quotidiennes, dans ses processus de changement et dans ses conséquences" (Lhotellier et Saint-Arnaud, 1994, p. 95) n'apparaît pas explicitement. Lorsque ce soucis d'articuler théorie et pratiques sociales du numérique est repérable, la posture du chercheur est le plus souvent de surplomb, avec l'élaboration de préconisations («nous proposons des préconisations pour un diagnostic automatique des erreurs des élèves dans le but de produire des rétroactions élaborées à partir du modèle praxéologique développé dans la thèse ») qui déplace le chercheur vers une posture d'expert ou de décideur (Rinaudo, 2017). On ne repère pas, à partir de l'analyse des résumés, de travail doctoral conduit selon une posture qui se rapprocherait d'un accompagnement du changement (Bedin, 2013) tandis que la conduite du changement semble plus présente: "proposer des pistes technologiques pour la conception et la mise en œuvre de dispositifs ».

Les contraintes de plus en plus pressantes liées à la durée de la thèse notamment, peuvent en partie expliquer la difficulté que peuvent avoir des doctorants à conduire des recherches dans une perspective d'accompagnement du changement qui nécessite un temps long, parfois incompatible avec les trois années de financement de la thèse, à moins que la thèse puisse s'inscrire dans un travail déjà amorcé par la direction de la thèse. Ce constat interroge sur la formation des jeunes chercheurs. Toutefois, il faut ici préciser que les données recueillies à partir des fichiers de la $70^{\circ}$ section du $\mathrm{CNU}$ ne permettent pas de mesurer la durée des thèses, pas plus que le mode de financement et le type de contrat. 
Dans un texte précédent j'écrivais que tout chercheur étant mobilisé, même si cela n'était pas toujours conscient, par un désir de changement (Rinaudo, 2018). Les jeunes docteurs dont nous observons ici les travaux, ne se désintéressent pas de la portée sociale de leurs travaux. Citons par exemple: «les apports des recherches dans le domaine du elearning dont nous pourrions bénéficier pour enrichir la pratique». Toutefois, plutôt qu'une visée praxéologique, d'articulation entre théorie et pratique, je soutiens l'idée que les recherches doctorales sur le numérique sont conduites, pour plusieurs d'entre elles, avec une visée pragmatique. Selon cette visée, la théorisation prend en compte le contexte de l'action, mais l'articulation entre théorie et pratique se déroule toujours dans une direction descendante de la théorie vers la pratique. La pratique ne doit donc résulter que d'une application, la plus juste possible, de la théorie. Contrairement à la praxéologie qui articule dans une même théorie pratique et action, la pragmatique propose une théorie pour l'action. Les préconisations à destination des acteurs sont un indice de cette orientation de recherche.

\section{Des paradoxes pour conclure}

Ce travail sur les résumés des thèses met au jour deux paradoxes.

Ainsi, dans la plupart des travaux de recherche sur le numérique menés par les doctorants, le changement proposé reste essentiellement une modification de surface, mais sans réelle modification en profondeur, alors même que ces recherches s'intéressent à des dispositifs innovants ou prétendus tels que constituent les outils du numérique. Parce que les chercheurs n'accompagnent généralement pas le changement dans une recherche avec les praticiens de l'éducation, on se trouve davantage en présence de modification que de métamorphose ou de transmutation pour reprendre les conceptions du changement en éducation que proposent Janner-Raimondi et Tavignot (2016). Les changements qui résultent de ces travaux semblent liés à la nouveauté des outils ou des dispositifs plutôt qu'à une transformation des pratiques des acteurs, éducateurs ou apprenants, de leur rapport au numérique et de leur rapport au savoir.

Le second paradoxe est que les recherches doctorales sur ces dispositifs, dans le champ de l'éducation et de la formation, sont menées selon des modalités voire des paradigmes de recherche, pour la plupart d'entre eux, traditionnels. Or, ceux qui deviendront chercheurs ou enseignants-chercheurs seront amenés à travailler, de plus en plus, dans des recherches commanditées pour lesquelles la recherche-intervention peut constituer une "clef d'entrée" (Bedin, 1993, p. 101) pour accompagner le changement dans les sphères éducatives. Ceci est particulièrement le cas pour les spécialistes du numérique avec les demandes et commandes des collectivités locales et des institutions sur les dispositifs qu'elles promeuvent et mettent en œuvre.

Il ne s'agit aucunement dans cet article de mettre en cause d'une quelconque façon le travail des docteurs durant leur thèse. Peut-être qu'un travail de recherche et d'accompagnement du changement auprès des praticiens constitue un impossible insurmontable dans le cadre du travail doctoral. Probablement parce que l'évaluation du travail de doctorat se constitue, quelles que soient les démarches et les orientations épistémologiques, sur la mise au jour d'une question problématisée, étudiée selon une méthodologie pertinente et rigoureuse, qui produit une analyse cohérente avec le cadre choisi et étayée par une littérature scientifique contemporaine et reconnue. Dit 
autrement, ce qui est demandé à un doctorant c'est de faire la preuve, tant lors de la soutenance que dans les publications scientifiques, de sa capacité à conduire une recherche de façon canonique. Or, l'intervention et l'accompagnement du changement demandent d'autres compétences et produisent d'autres effets et qui ne sont pas considérés au même titre que la production d'un savoir scientifique. Sans doute également, et on revient sur les contraintes de temps déjà évoquées, parce que les changements de pratiques impulsées ou révélées par le numérique sont-elles davantage inscrites dans la durée que dans des temporalités d'immédiateté auxquelles nous confrontent les technologies numériques (Musso, 2009).

\section{BIBLIOGRAPHIE}

Baron, GL. (2013). La recherche francophone sur les « technologies » en éducation : réflexions rétrospectives et prospectives. Sticef, 20, en ligne : http://sticef.univ-lemans.fr/num/vol2013/16baron-reiah/sticef_2013_NS_baron_16p.pdf (consulté en avril 2020).

Bedin, V. (2013). La recherche-intervention en éducation et en formation : une nouvelle forme de conduite et d'accompagnement du changement. in V. Bedin (coord.). Conduite et accompagnement du changement. Contribution des sciences de l'éducation (p. 87-105). Paris : L'Harmattan.

Beillerot, J. (1991). La recherche : essai d'analyse. Recherche et formation, 9, 17-31.

Beillerot, J. (1993). Les thèses en sciences de l'éducation. Bilan de vingt années d'une discipline : 19691989. Rapport de recherche. Nanterre : Université Paris 10.

Beillerot, J. \& Demori, F. (1998). Les thèses de sciences de l'éducation de 1990 à 1994. Bulletin de l'association des enseignants et chercheurs en sciences de l'éducation, 20-21, 51-93.

Chevallard, Y. (1998). Analyse des pratiques enseignantes et didactique des mathématiques : l'approche anthropologique. Actes de l'université d'été IREM (p. 91-119). IREM Clermont-Ferrand.

Dessus, P. (1999). Où va la recherche en éducation? Analyse factorielle de résumés de communications aux Biennales de l'éducation et de la formation. L'année de la recherche en sciences de l'éducation, 6, 201-219.

Durkheim, E. (1922). Education et sociologie. Paris : PUF, 2013.

Heiden, S., Magué, JP., Pincemin, B. (2010). TXM : Une plateforme logicielle open-source pour la textométrie. Conception et développement. In I. C. Sergio Bolasco (Éd.). 10th International Conference on the Statistical Analysis of Textual Data - JADT 2010 (Vol. 2, p. 1021-1032). Edizioni Universitarie di Lettere Economia Diritto. En ligne : http://halshs.archives-ouvertes.fr/ halshs-00549779/en/ (consulté en avril 2020)

Janner-Raimondi, M., Tavignot, P. (2016). La notion de changement en éducation et formation : entre approche plurielle et regards croisés. in J-L. Rinaudo, P. Tavignot (dir.). Le changement à l'école. Sources, tensions, effets (p. 15-40). Paris : L'Harmattan.

Lhotellier, A., Saint-Arnaud, Y. (1994). Pour une démarche praxéologique. Nouvelles pratiques sociales, 7 (2), 93-109. 
Musso, P. (2009). Usages et imaginaires des TIC : la fiction des frictions. In C. Licoppe (dir). L'évolution des cultures numériques, de la mutation du lien social à l'organisation du travail (p. 201-210). Limoges : FYP.

Rinaudo, JL. (2017). Postures de recherche et émancipation. in J-F. Marcel, D. Broussal (dir.). Émancipation et recherche en éducation (p. 377-395). Vulaines sur Seine : Eds du Croquant.

Rinaudo, JL. (2018). Changement et recherche en éducation : quelques paradoxes. in D. Broussal, K. Bonnaud, JF. Marcel, P. Sahuc (dir.). Recherche(s) et changement(s) : dialogues et relations (p. 207-214). Toulouse : Cépaduès Editions.

Rinaudo, JL. (2019). Les recherches participatives en sciences de l'éducation : point de vue du CNU. Colloque Hommage à François Villemonteix. Université Paris Descartes, 19 mars.

Saint-Arnaud, Y., Mandeville, L., Bellemare, C. (2002). La praxéologie. Interactions 6, 1, 29-48.

\section{RÉSUMÉS}

L'auteur propose une étude des résumés de thèse portant sur le numérique des docteurs ayant présenté un dossier de qualification aux fonctions de maitre de conférences, auprès de la $70^{\mathrm{e}}$ section du CNU, entre 2015 et 2019. L'analyse est conduite à partir du lexique employé dans ces résumés. La visée heuristique est présente, la visée critique rare. Quant à la visée praxéologique, l'auteur propose qu'elle soit effacée au profit d'une visée pragmatique.

The author proposes a study of the abstracts of thesis on the digital of the doctors who submitted a dossier of qualification to the functions of lecturer, at the 70th section of the CNU, between 2015 and 2019. The analysis is conducted from the lexicon used in these abstracts. The heuristic focus is present, the critical focus rare. As for the praxeological aim, the author proposes that it be erased in favour of a pragmatic aim.

\section{INDEX}

Mots-clés : doctorat, numérique, analyse lexicométrique, résumés de thèse

Keywords : PhD, digital, lexicometric analysis, abstracts

\section{AUTEUR \\ JEAN-LUC RINAUDO}

Professeur en sciences de l'éducation, Université de Rouen-Normandie, Cirnef EA 7454 